\title{
Dynamics of Fluid Vesicles in Flow through Structured Microchan- nels
}

\author{
Hiroshi Noguchi ${ }^{1,2}$ (a) ${ }^{\text {(a) Gerhard Gomprer }}{ }^{1}$ (b), Lothar Schmid $^{3}$ (c), Achim Wixforth ${ }^{3}$, and Thomas \\ FrANKE $^{3}$ (d) \\ 1 Institut für Festkörperforschung, Forschungszentrum Jülich, 52425 Jülich, Germany, \\ 2 Institute for Solid State Physics, University of Tokyo, Kashiwa, Chiba 277-8581, Japan, \\ 3 Experimentalphysik I, Microfluidics Group, Universität Augsburg, 86159 Augsburg, Germany
}

PACS 87.16.D- - Membranes, bilayers, and vesicles

PACS 83.50. Ha - Flow in channels

PACS 82.70.Uv - Surfactants, micellar solutions, vesicles, lamellae, amphiphilic systems

\begin{abstract}
The dynamics of fluid vesicles is studied under flow in microchannels, in which the width varies periodically along the channel. Three types of flow instabilities of prolate vesicles are found. For small quasi-spherical vesicles - compared to the average channel width - perturbation theory predicts a transition from a state with orientational oscillations of a fixed prolate shape to a state with shape oscillations of symmetrical ellipsoidal or bullet-like shapes with increasing flow velocity. Experimentally, such orientational oscillations are observed during the slow migration of a vesicle towards the centerline of the channel. For larger vesicles, mesoscale hydrodynamics simulations and experiments show similar symmetric shape oscillation at reduced volumes $V^{*} \gtrsim$ 0.9 . However, for non-spherical vesicles with $V^{*} \lesssim 0.9$, shapes are found with two symmetric or a single asymmetric tail.
\end{abstract}

Introduction. - Soft deformable objects such as liquid droplets, vesicles, and cells show a complex behavior under flow. For example, in simple shear flow, fluid vesicles exhibit tank-treading, tumbling, and swinging (also called vacillating-breathing, or trembling) motions, de' pending on parameters such as shear rate, viscosity contrast, and internal volume 119. Understanding the flow - behavior of lipid vesicles and red blood cells (RBCs) is not only an interesting problem of the hydrodynamics of deformable, thermally fluctuating membranes, but is also important for medical applications. In microcirculation, the deformation of RBCs reduces the flow resistance of microvessels. In diseases such as sickle cell anemia, RBCs have reduced deformability and often block microvascular flow [10. Lipid vesicles are considered as a simple model of RBCs and also have applications as drug-delivery systems.

The recent development of microfluidic techniques [11] allows the investigation and manipulation of individual

\footnotetext{
(a) E-mail:noguchi@issp.u-tokyo.ac.jp

(b) E-mail:g gompper@fz-juelich.de

(c) E-mail:lothar.schmid@physik . uni-augsburg.de

(d) E-mail:thomas franke@physik . uni-augsburg. de
}

cells and vesicles, e.g. the measurement of the dynamic pressure drop for single-cell deformation [12, the separation of RBCs from the suspending plasma [13, 14, and the control of oxygen concentration to investigate sickle cells 10. There are many potential applications such as blood diagnosis on chip. Under steady flows in homogeneous glass capillaries and rectangular microchannels, vesicles [15] and RBCs [14, 16, 18, deform into a bullet (with a flattened rear end) or parachute (with an inside bulge at the rear end) shape. Compared to these steadyflow conditions, the vesicle dynamics in time-dependent flow is much less explored. Only very recently, phenomena like the wrinkling of vesicles after inversion of an elongational flow [19,20] or shape oscillation of RBCs [21,22 and fluid vesicles 23, under oscillatory shear flow have been discovered.

In this paper, we propose a structured microchannel system to study vesicle dynamics. The width of a microchannel is spatially modulated along the channel, so that a flowing vesicle is exposed to an oscillatory elongational flow. We have fabricated such microchannels and observe the time-dependent vesicle deformation via optical microscopy. In parallel, we use perturbation theory 
for quasi-spherical vesicles and mesoscale-hydrodynamics simulations for non-spherical vesicles to predict several flow instabilities. In particular, we predict for wider channels (compared to the vesicle size) a transition from shape oscillations of symmetrical bullet-like shapes to orientational oscillations with decreasing flow velocity; for narrower channels, we observe stable shapes with two symmetric or with a single asymmetric tail. Our results demonstrate that structured channels are well suited to investigate the dynamical behavior of vesicles.

Experimental Systems and Methods. - Lipid vesicles, consisting of 1,2-Dioleoyl-sn-Glycero-3Phosphocholine (DOPC, Avanti Polar Lipids) with $0.1 \mathrm{~mol} \%$ fluorescently labeled T-Red DHPE (Texas Red 1,2-dihexadecanoyl-sn-glycero-3-phosphoethanolamine, triethylammonium salt, Invitrogen, USA), were prepared in aqueous sucrose solution $(200 \mathrm{mM})$ by electroformation, as described elsewhere 24. Using this method, many giant unilamellar vesicles with diameter larger than $10 \mu \mathrm{m}$ were produced. Prior to the experiments, the vesicles were introduced into an slightly hypertonic aqueous glucose or sucrose solution. This procedure enables control of the volume-to-surface ratio by inflation and deflation of the vesicles. The vesicles in sucrose solution are perfectly density matched. The density difference between sucrose and glucose solutions at $200 \mathrm{mM}$ is $\Delta \rho / \rho=0.012$, which implies a small buoyancy force.

Microchannels were fabricated by soft lithography 30 . The structure of an AutoCAD designed mask was transferred by illumination to a photoresist (SU-8, microresist, Berlin), which serves as a master to cast poly(dimethylsiloxane) (PDMS) replicas. After treatment with an $\mathrm{O}_{2}$-plasma, the PDMS mold was bonded onto a thin coverslip. The assembly was connected to a reservoir containing the vesicle solution (inlet) and a water reservoir (outlet) to adjust the hydrostatic pressure difference, which drives the fluid through the structured microchannel (see Fig. 1). All channels have a height $L_{z}=50 \mu \mathrm{m}$ and a periodic length $L_{x}=100 \mu \mathrm{m}$.

Due to the lift force from a wall 25+27 and the migration in a parabolic Poiseuille flow 28, 29, vesicles are aligned close to the center of the channel. In the glucose solutions, the buoyancy force leads to a displacement of the vesicles from the center of the channel in the $z$-direction (see Fig. 1). The balance of the buoyancy force and the lift force [25, 27. implies a distance $\ell \simeq\left(\eta \dot{\gamma} R_{0} /(\Delta \rho g)\right)^{1 / 2}$ between membrane and wall, where $\dot{\gamma}$ is an effective shear rate, $R_{0}$ a mean vesicle radius, $\eta$ the fluid viscosity, and $g$ the gravitational acceleration. For $R_{0}=10 \mu \mathrm{m}, \dot{\gamma}=1 \mathrm{~s}^{-1}$ (corresponding to a mean flow velocity of about $20 \mu \mathrm{m} / \mathrm{s}$ ) and the viscosity of water, we obtain the order-of-magnitude estimate of $\ell=10 \mu \mathrm{m}$, comparable to the channel size $L_{z}$. The displacement from the center implies an asymmetric deformation of the vesicle shape in glucose solution under flow.

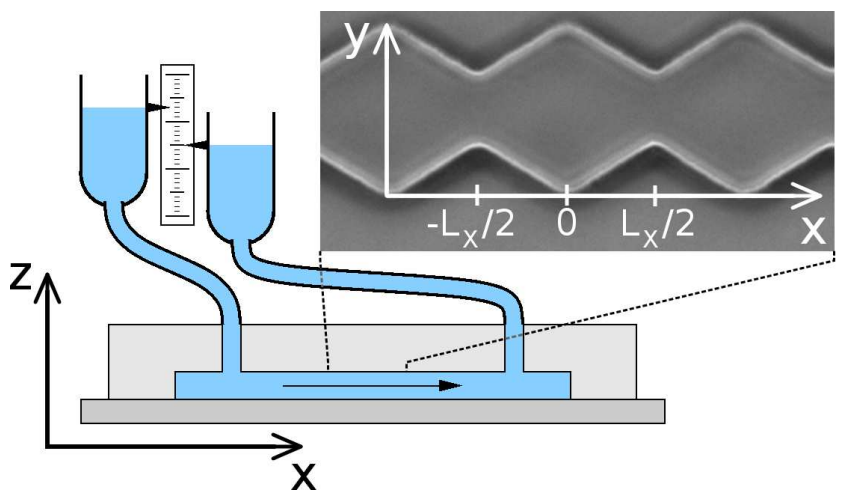

Fig. 1: (Color online) Side view of the channel setup with the reservoirs. The inset shows a top view of the modulated channel. The length of the modulation is $L_{x}=100 \mu \mathrm{m}$.

Theory for quasi-spherical vesicles in modulated channels. - We consider vesicles which have the same viscosity $\eta$ of the outer and inner fluids. Vesicles have constant volume $V$ and constant surface area $S$, so that the reduced volume $V^{*}$ and the excess area $\Delta_{\mathrm{S}}$ are defined by $V^{*}=\left(R_{\mathrm{V}} / R_{\mathrm{S}}\right)^{3}=\left(1+\Delta_{\mathrm{S}} / 4 \pi\right)^{-3 / 2}$ and $\Delta_{\mathrm{S}}=S / R_{\mathrm{V}}^{2}-$ $4 \pi$, where $R_{\mathrm{V}}=(3 V / 4 \pi)^{1 / 3}$ and $R_{\mathrm{S}}=(S / 4 \pi)^{1 / 2}$.

First, we derive an analytical description of the flow behavior of small quasi-spherical vesicles with $\Delta_{\mathrm{S}} \ll 1$, based on the theory for linear flows 2, 5, 7, 20. The microchannel has a constant height $L_{z}$ and a periodically modulated width with walls at $\pm h_{y}(x)$, where

$$
h_{y}(x)=\frac{L_{y}}{2}\left\{1+a_{y} \cos \left(\frac{2 \pi x}{L_{x}}\right)\right\} .
$$

Here, $L_{x}$ is the periodicity length along the channel (see Fig. 11). The flow field $\mathbf{v}(\mathbf{r})$ for weakly modulated channels $\left(a_{y} \ll L_{x} / L_{y}\right)$ is obtained from lubrication theory,

$$
\begin{aligned}
& v_{x}=\left(9 v_{\mathrm{m}} L_{y} / 8 h_{y}\right)\left\{1-\left(y / h_{y}\right)^{2}\right\}\left\{1-\left(2 z / L_{z}\right)^{2}\right\}, \\
& v_{y}=-\pi v_{x} y L_{y} a_{y} \sin \left(2 \pi x / L_{x}\right) / h_{y} L_{x}, \\
& v_{z}=0,
\end{aligned}
$$

where $v_{\mathrm{m}}$ is the mean flow velocity. Furthermore, we consider small vesicles with $R_{\mathrm{V}} \ll L_{y}, L_{z}$, and neglect backflow effects due to the channel wall.

The vesicle shape is expanded in spherical coordinates as $r=R_{\mathrm{V}}\left(1+\sum_{l, m} u_{l, m} Y_{l, m}\right)$, with the spherical harmonics $Y_{l, m}(\theta, \varphi)$ and the polar axis in the $z$ direction. We consider a vesicle shape which is mirror symmetric in the $z$ direction, which implies, e.g., $u_{2, \pm 1}=u_{3,0}=u_{3, \pm 2}=0$. The curvature energy of the membrane with bending rigidity $\kappa$ and reduced surface tension $\sigma$ is given by

$$
F=(\kappa / 2) \sum_{l, m} E_{l}\left|u_{l, m}\right|^{2}+\kappa(12+2 \sigma / 3) u_{2}^{(3)},
$$

where $E_{l}=(l+2)(l-1)\{l(l+1)+\sigma\}$. Here, the third order term $u_{2}^{(3)}=(\sqrt{5 / \pi} / 7)\left(u_{2,0}{ }^{3}-6 u_{2,0}\left|u_{2,2}\right|^{2}\right)$ for $l=2$ is necessary to obtain a prolate shape as thermal-equilibrium 
state [7,31]. With the Stokes approximation and the Lamb solution for the flow field, the dynamics of the amplitudes $u_{l, m}$ of a vesicle moving along the center line $(y=z=0)$ of the channel with velocity $\mathbf{v}\left(x_{\mathrm{G}}, 0,0\right)=\left(9 v_{\mathrm{m}} L_{y} / 8 h_{y}, 0,0\right)$ is governed by [2, 7, 20,

$$
\frac{\partial u_{l, m}}{\partial t}=s_{l, m}\left(x_{\mathrm{G}}\right)-\kappa^{*} \Gamma_{l} f_{l, m}
$$

with reduced bending rigidity $\kappa^{*}=\kappa L_{y} /\left(\eta R_{\mathrm{V}}{ }^{3} v_{\mathrm{m}}\right)=$ $L_{y} /\left(v_{\mathrm{m}} \tau\right)$ and $\Gamma_{l}=l(l+1) /(2 l+1)\left(2 l^{2}+2 l-1\right)$. Here, $\tau=\eta R_{\mathrm{V}}^{3} / \kappa$ is the characteristic relaxation time of a vesicle. Two kinds of forces determine the membrane deformation in Eq. (4), the curvature force $f_{l, m}=E_{l} u_{l, m}-$ $(12+2 \sigma / 3) \partial u_{2}^{(3)} / \partial u_{l, m}$, and the force $s_{l, m}\left(x_{\mathrm{G}}\right)$ due to the elongational flow. The force $s_{l, m}\left(x_{\mathrm{G}}\right)$ is given by $s_{l, m}\left(x_{\mathrm{G}}\right)=\left(a_{l, m}+B_{l} b_{l, m}\right) L_{y} / R_{\mathrm{V}} v_{\mathrm{m}}$ [2, 2, 20, Here, $B_{l}=$ $1 /\left(2 l^{2}+2 l-1\right)$, and $a_{l, m}$ and $b_{l, m}$ are the spherical harmonics expansion of $v_{r}(\mathbf{r})$ and $R_{\mathrm{V}} \partial v_{r}(\mathbf{r}) / \partial r$ at $\left|\mathbf{r}-\mathbf{r}_{\mathrm{G}}\right|=R_{\mathrm{V}}$, respectively, where $v_{r}(\mathbf{r})$ is the radial components of the flow field $\mathbf{v}(\mathbf{r})$ in the absence of the vesicle. The tension $\sigma$ is determined by the area constraint $\partial \Delta_{\mathrm{S}} / \partial t=0$ with $\Delta_{\mathrm{S}}=\sum_{l, m}(l+2)(l-1)\left|u_{l, m}\right|^{2} / 2-2 u_{2}^{(3)} / 3$. We consider a vesicle with a small excess area $\Delta_{\mathrm{S}}=0.1$ (corresponding to $V^{*}=0.988$ ), where it is sufficient to take into account $l=2,3$ modes, and a channel with $L_{y}=L_{z}=20 R_{\mathrm{V}}$ and various $L_{x}$ and $a_{y}$.

In fast flow, where flow forces dominate and the reduced bending rigidity is small, $\kappa^{*} \ll 1$, the analysis of Eq. (4) shows that the shape is mirror symmetric with respect to both $x z$ and $x y$ planes, and that the vesicle lengths $l_{x}$ and $l_{y}$ oscillate (see Fig. 22). The flow elongates the vesicle in the $y$ and $x$ direction for $-L_{x} / 2<x<0$ and $0<x<L_{x} / 2$, respectively. In the regime $\kappa^{*} \ll 1$, the extremal elongations are determined by the balance of the flow forces, $s_{l, m}\left(x_{\mathrm{G}}\right)$, and the area constraint. The forces $\left|s_{2,2}\left(x_{\mathrm{G}}\right)\right|$ and $\left|s_{3,3}\left(x_{\mathrm{G}}\right)\right|$ have maxima at $x_{\mathrm{G}}= \pm 0.38 L_{x}$ and $x_{\mathrm{G}}= \pm L_{x} / 2$ for $a_{y}=0.5$, respectively. The resulting positions of extremal elongations are found to be close to $x_{\mathrm{G}}=0$ and $x_{\mathrm{G}}=L_{x} / 2$ (see Fig. 2(b)).

In contrast, in slow flow with $\kappa^{*} \gg 1$, the vesicle is predicted not to change its shape, but to display a periodic oscillation of its orientation. The tilt angle $\theta$ oscillates around $\pi / 4$ (or $-\pi / 4$ depending on initial positions), see snapshot in Fig. 2(a). Thus, a symmetry breaking occurs with decreasing flow velocity.

Both types of vesicle motions are limit cycles. A sharp but continuous transition between these two motions occurs at a critical reduced bending rigidity $\kappa_{\mathrm{c}}^{*}$, where the tilt angle $\theta$ vanishes (see Fig. 2(a)). The critical value $\kappa_{\mathrm{c}}^{*}$ increases with increasing corrugation $a_{y}$ of the channel, but is almost independent of $L_{x}$ for $L_{x} / L_{y}>2$ (see Fig. 2(c)). The symmetric vesicle deformation reduces the disturbance of the original flow but increases the free energy of a vesicle. At small or large $\kappa^{*}$, the former or latter contribution dominates, respectively.
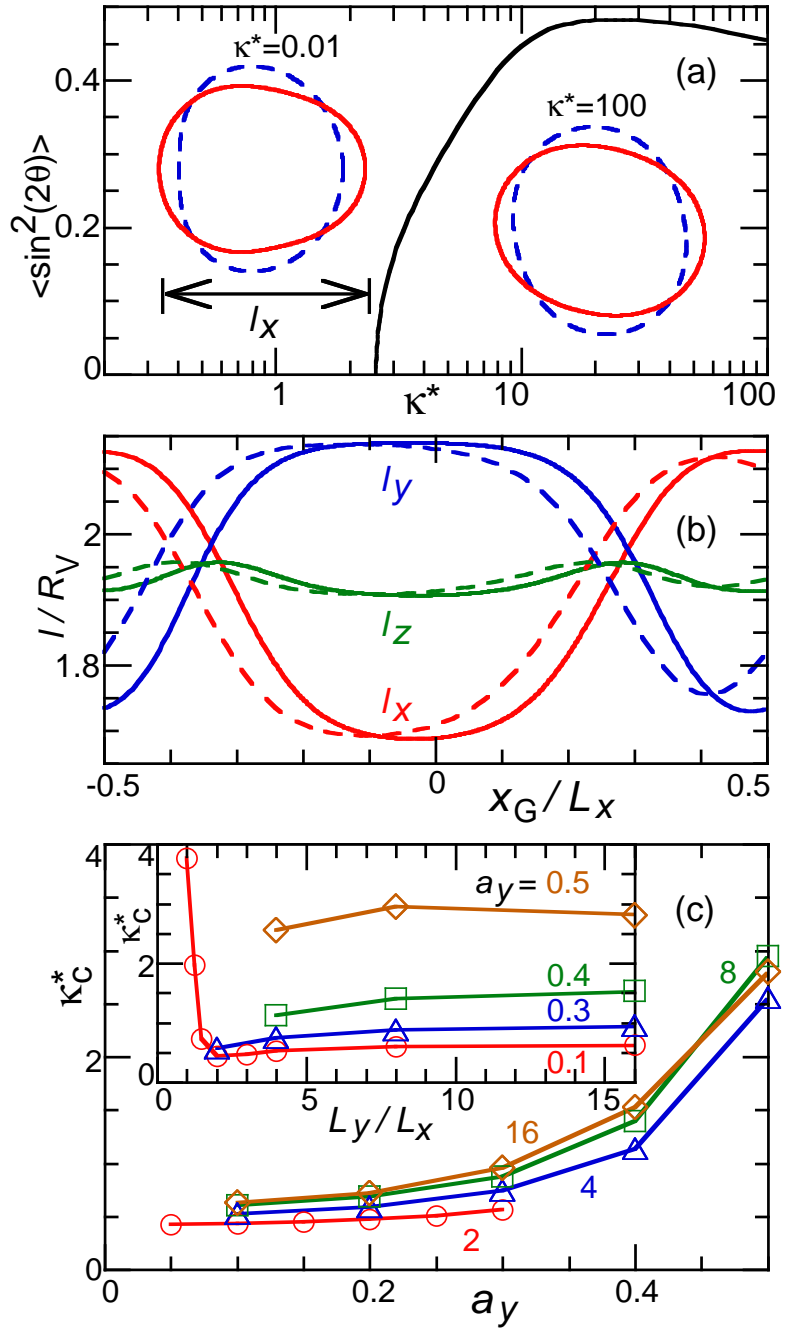

Fig. 2: (Color online) Dynamics of a quasi-spherical vesicle at $V^{*}=0.988$. (a) Dependence of the tilt angle $\theta$ of a vesicle on $\kappa^{*}$, for $L_{x} / L_{y}=4$ and $a_{y}=0.5$. Here, $\left\langle\sin ^{2}(2 \theta)\right\rangle=\left\langle\left(\operatorname{Im}\left(u_{22}\right) /\left|u_{22}\right|\right)^{2}\right\rangle$ describes the deviation from symmetric shape (with respect to the $x z$ plane). The insets show sliced snapshots of vesicles in the $x y$ plane for $\kappa^{*}=0.01$ and $\kappa^{*}=50$. Solid and dashed lines indicate shapes of extremal elongation or tilt. (b) Maximum vesicle lengths in the $x, y, z$ directions as a function of the center-of-mass position $x_{\mathrm{G}}$, for $\kappa^{*}=0.01$ and $a_{y}=0.5$. Solid and dashed lines represent results for $L_{x} / L_{y}=4$ and $L_{x} / L_{y}=16$, respectively. (c) Critical reduced bending rigidity $\kappa_{\mathrm{c}}^{*}$ as a function of the corrugation amplitudes $a_{y}$ for channel geometry $L_{x} / L_{y}=2$, 4,8 , and 16. The inset shows $\kappa_{\mathrm{c}}^{*}$ as a function of $L_{x} / L_{y}$ for $a_{y}=0.1,0.3,0.4$, and 0.5 .

Mesoscale Hydrodynamics Simulations of Vesicles under Flow. - We employ mesoscale hydrodynamics simulations to study the flow behavior of vesicles in narrow channels. A dynamically-triangulated surface model for the membrane 32 is combined with a particle-based mesoscale simulation technique - multi-particle collision dynamics (MPC) 33 35 — for the embedding fluid. A detailed description of this approach to model vesicles dy- 


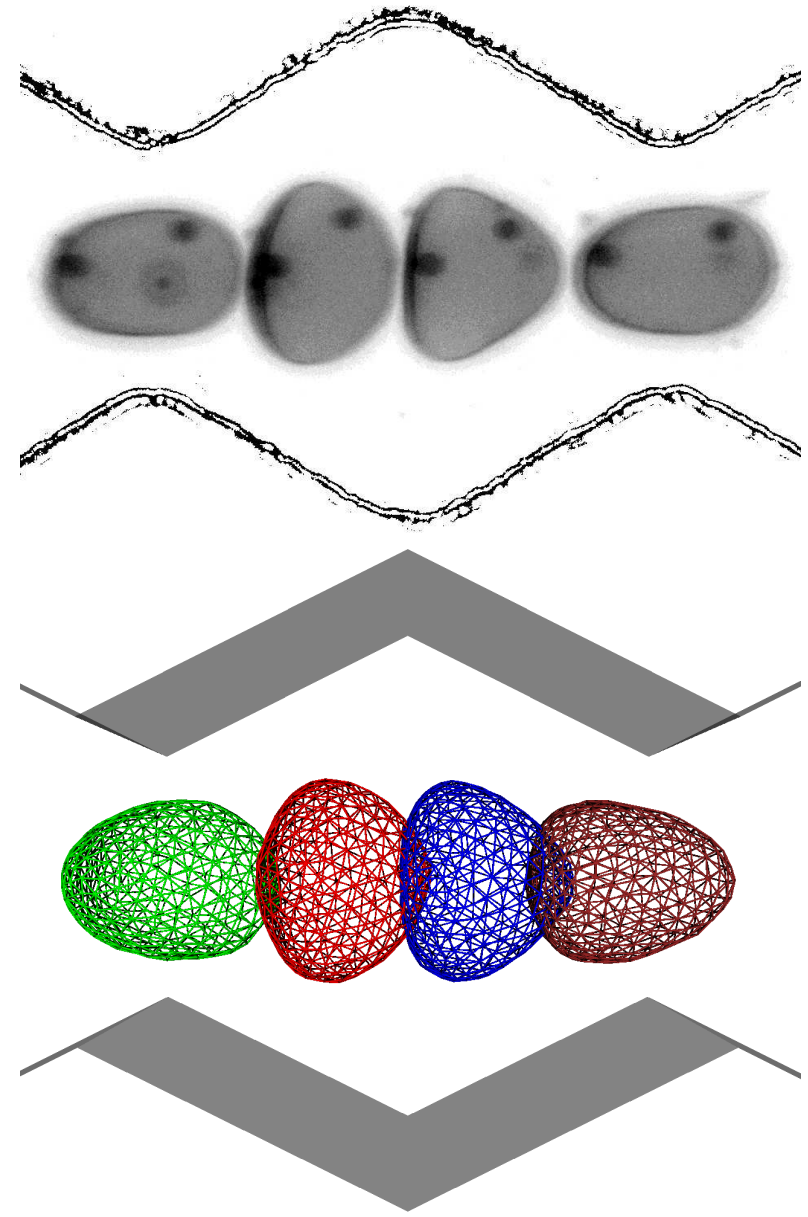

Fig. 3: (Color online) Sequence of snapshots (at equal time intervals) of vesicles with $V *=0.96$ and $R_{\mathrm{S}} / L_{y}=0.21$ moving through a microchannel, experimentally observed by optical microscopy (in sucrose solution) for $\kappa^{*}=0.04\left(v_{\mathrm{m}}=38 \mu \mathrm{m} / \mathrm{sec}\right.$, $L_{y}=75 \mu \mathrm{m}$ ) (top), and in simulation for $\kappa^{*}=0.08$ (bottom). For the experimental data, the time interval between images is $0.97 \mathrm{sec}$.

namics under flow can be found in Refs. 4, 18. In MPC, the fluid is described by $N_{\mathrm{s}}$ point-like particles of mass $m_{\mathrm{s}}$. After free streaming of every particle for a time step $\Delta t$, the fluid particles collide in cubic boxes of lattice constant $a=L_{x} / 16$, which is also the mean length between membrane vertices. We use the fluid viscosity $\eta=550 \sqrt{m_{\mathrm{s}} k_{\mathrm{B}} T} / a^{2}$ (with number density $n=100 a^{-3}$ and the time step $\left.\Delta t=0.01 a \sqrt{m_{\mathrm{s}} / k_{\mathrm{B}} T}\right)$, corresponding to low Reynolds numbers, to simulate experimental conditions. We study vesicles with bending rigidity $\kappa=20 k_{\mathrm{B}} T$ where $k_{\mathrm{B}} T$ is the thermal energy. The flow velocity is chosen to be $v_{\mathrm{m}} \tau / L_{y}=170\left(R_{\mathrm{V}} / L_{y}\right)^{3}$ for a channel with $L_{y}=L_{z}=L_{x} / 2$, corresponding to a slow vesicle relaxation compared to the passage time through a channel segment. For vesicles with $R_{\mathrm{S}} / L_{y}=0.36$ and $R_{\mathrm{S}} / L_{y}=0.46$ at $V^{*}=0.9$, the reduced bending rigidity is $\kappa^{*}=0.14$ and $\kappa^{*}=0.07$, respectively. In this regime, the vesicle dynamics is not very sensitive to $\kappa^{*}$.

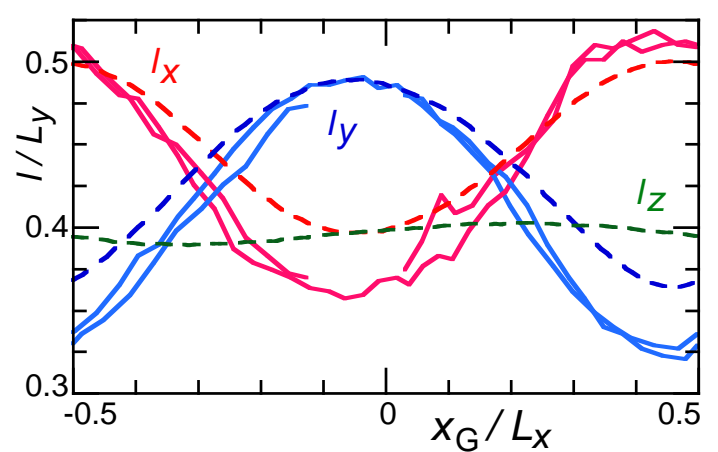

Fig. 4: (Color online) Maximum lengths $l_{x}, l_{y}$, and $l_{z}$ of vesicles in experiments (solid lines) and simulations (dashed lines) from the same data as shown in Fig. 3 .

Vesicles in sawtooth-shaped channels in fast flows. - We investigate the dynamics of large prolate vesicles in fast flows, corresponding to small $\kappa^{*}$, both by experiments and simulations. In this case, hydrodynamic interactions between the vesicle and wall are not negligible. We consider now a periodically-patterned microchannel with a saw-tooth shape (see Figs. 11 and 3), for which the walls are located at

$$
h_{y}(x)=\frac{L_{y}}{2}\left\{1+a_{y}\left(1 \pm \frac{4 x}{L_{x}}\right)\right\}
$$

for $-L_{x} / 2<x \leq 0$ and $0<x \leq L_{x} / 2$, respectively. Two types of channels are used, which are characterized by $L_{y}=50 \mu \mathrm{m}, a_{y}=0.2$ and $L_{y}=75 \mu \mathrm{m}, a_{y}=0.33$ (as well as $L_{z}=50 \mu \mathrm{m}$ and $L_{x}=100 \mu \mathrm{m}$ ). The glucose and sucrose solutions are used for the experiments with the narrower and wider channels, respectively. Typical experimental flow velocities are in the range $v_{\mathrm{m}}=10-$ $100 \mu \mathrm{m} / \mathrm{s}$.

First, we describe the dynamics in the wider channel $\left(L_{y}=75 \mu \mathrm{m}\right)$. Experimental and simulation results for the shape deformation are shown in Figs. 3 and 4 . As expected, the vesicles change their shapes periodically. For $V^{*} \gtrsim 0.9$, vesicle shapes vary continuously between an almost ellipsoidal (in the narrow part of the channel) and a cone-like (in the wide part of the channel) bullet shape, see Fig. 3. This is in contrast to the behavior for unbounded steady Poiseuille flow, where perturbation theory predicts a coexistence between bullet- and parachute-like shapes at the center line 29. The amplitudes and phases of the deformations in the $x$ - and $y$-directions (see Fig. (4) agree well between simulations and experiments, while very little deformation is seen in the $z$-direction. The small difference of amplitudes between the experiment and simulation is probably due to the somewhat different values of the reduced volume and radius of the vesicle, since an accurate experimental estimation is difficult (because the length in the $z$ direction could not be measured). The dynamics also agrees qualitatively with the theoretical predictions for quasi-spherical vesicles, compare Fig. 2, although the parameters are outside the range of validity of the theo- 
Dynamics of Fluid Vesicles in Flow through Structured Microchannels

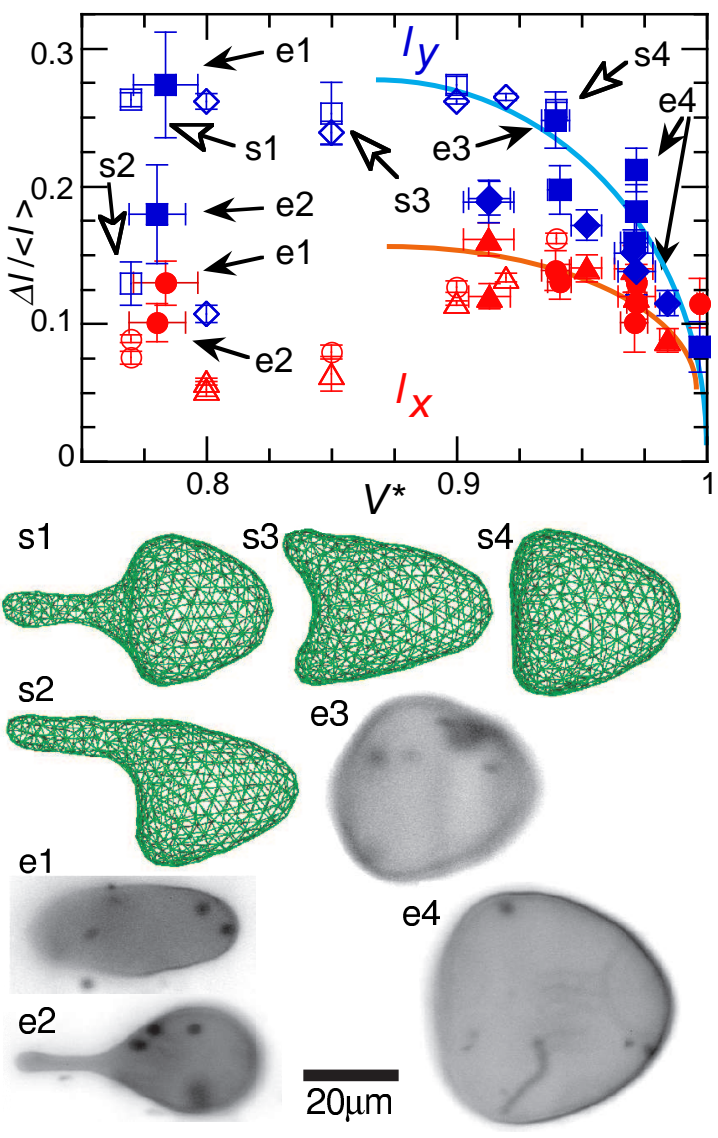

Fig. 5: (Color online) Normalized oscillation amplitudes $\Delta l /\langle l\rangle$ in the $x$ (red) and $y$ (blue) directions. Open symbols represent simulation data for $R_{\mathrm{S}} / L_{y}=0.36(\circ, \square)$ and $R_{\mathrm{S}} / L_{y}=0.46$ $(\triangle, \diamond)$ at $a_{y}=0.2$, respectively. Closed symbols represent experimental data for $L_{y}=50 \mu \mathrm{m}$ with $a_{y}=0.2$ in glucose solution $(\bullet, \boldsymbol{\square})$ and $L_{y}=75 \mu \mathrm{m}$ with $a_{y}=0.33$ in sucrose solution $(\boldsymbol{\Delta}, \mathbf{\bullet})$, respectively. The solid lines are guide to the eye. The lower part shows snapshots of simulations (s1, s2, s3, s4) and experiments (e1, e2, e3, e4) for $L_{y}=50 \mu \mathrm{m}$. All snapshots are displayed with the same length scale. The pictures e1 and e2 show the same vesicle before and after the transformation from ellipsoid to tailed shape, respectively. All images (e1-e4) are vesicles in glucose solution.

retical approximations.

At reduced volumes $V^{*} \lesssim 0.9$, we find that the vesicles deform into novel shapes, while they are still ellipsoidal in the absence of flow. At $V^{*} \sim 0.85$, the simulated vesicles develop two tails in the $x y$ plane (see Fig. 囵(s3)). This tail position is very stable; the tails quickly return to the $x y$ plane, even from an initial state with the tails in $x z$ plane (obtained by $\pi / 2$ rotation). At $V^{*} \sim 0.80$, the symmetric tails become unstable; they are replaced by a stable shape with a single asymmetric tail (see Fig. 囵(s2)). In this case, the configuration with a tail in the $x z$ plane is also stable within the accessible simulation time (see Fig. 5 (s1)). A single-tail shape is also observed in our experiments with

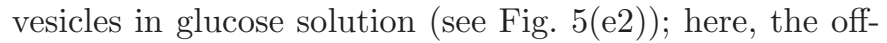
center motion induced by the buoyancy force implies that

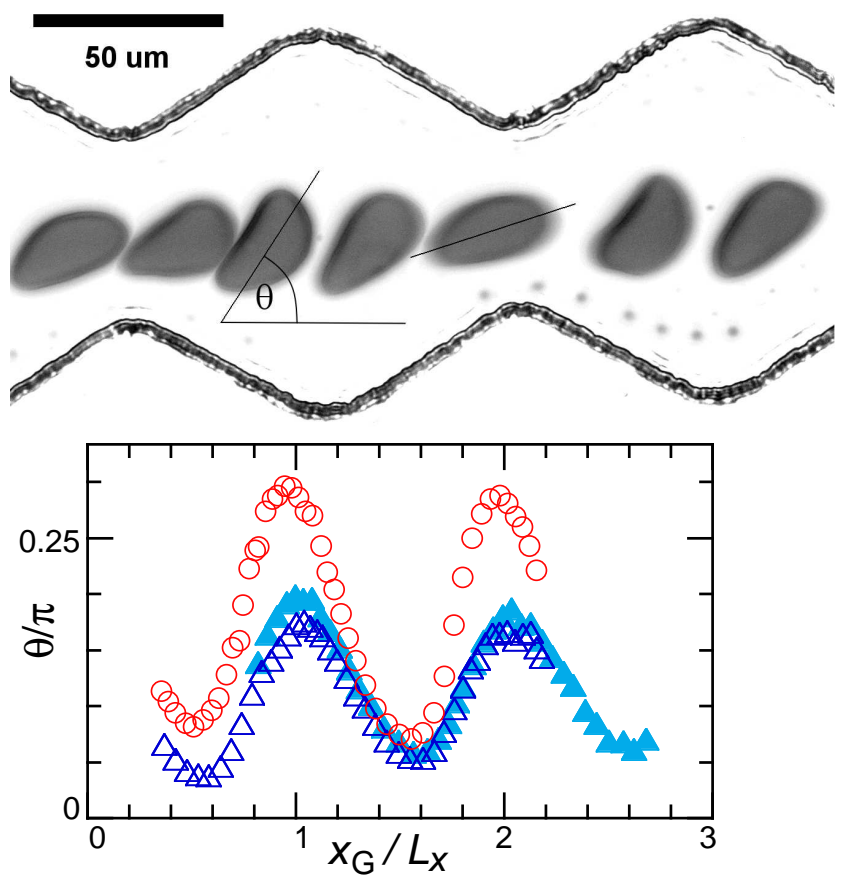

Fig. 6: (Color online) Oriental oscillation of a vesicle (in sucrose solution) in a wide structured channel with $L_{y}=75 \mu \mathrm{m}$. (a) Micrograph of an oscillating vesicle with $R_{\mathrm{V}}=24 \mu \mathrm{m}$, $v=34 \mu \mathrm{m} / \mathrm{s}$, corresponding to $\kappa^{*}=0.0132$. The image is a superposition of video frames taken at time intervals of $1 \mathrm{~s}$. (b) Orientational angle as a function of the center-of-mass position $x_{\mathrm{G}}$. The (red) circles (o) represent the same data as in (a). The vesicle oscillates between the extrema of $\theta_{\min }=0.07 \pi$ and $\theta_{\max }=0.3 \pi$. The (blue) triangles represent another vesicle (with $R_{\mathrm{V}}=18 \mu \mathrm{m}, v= \pm 55 \mu \mathrm{m} / \mathrm{s}$ ). Here, we have inverted the direction of the fluid flow; symbols indicate angles before $(\triangle)$ and after $(\Delta)$ flow inversion.

the tail is found in the $z$ direction. Recently, Abkarian et al. 14 found a similar asymmetric tail for RBCs in very fast flows in homogeneous capillaries (with flow velocity $v_{m}=10-35 \mathrm{~cm} / \mathrm{s}$ and capillary diameter $\left.10 \mu \mathrm{m}\right)$. Such a long tail is much more difficult to form in RBCs due to the shear resistance of the spectrin network, and may even require a local separation of the lipid bilayer from the spectrin network. Thus, the formation of an asymmetric long tail is a generic feature in capillary flows. Structured channels promote tail formation already at smaller flow velocities. The low contrast and the blurry contour in the rear part of the vesicles in the experimental micrographs of Fig. 5 (e3, e4) indicates that vesicles exhibit enhanced shape fluctuations at the rear part, due to a locally reduced membrane tension; the low contrast regions in the shapes of Fig. 5 (e1, e2) is probably due to a tilt of the vesicle axis and a vesicle asymmetry induced by off-center motion with a buoyancy force.

Our experimental and simulation results for the oscillation amplitude $\Delta l$, displayed in top part of Fig. 5 , show that the difference between maximum and minimum of 
$l_{x}$ or $l_{y}$ is quite insensitive to the reduced volume $V^{*}$ and the vesicle size $R_{\mathrm{S}}$. In the experiments, $V^{*}$ is estimated from the prolate ellipsoid shape with the lengths $\left\langle l_{x}\right\rangle$ and $\left\langle l_{y}\right\rangle$ of the long and the two short axes, respectively. The finite amplitudes for $V^{*} \simeq 1$ are caused by errors in the estimation of $V^{*}$. The velocity $v_{\text {ves }}$ and average size $l_{\mathrm{m}}=\left(\left\langle l_{x}\right\rangle+\left\langle l_{x}\right\rangle\right) / 2$ of liposomes are varied in experiments in the ranges $v_{\text {ves }}=14-45 \mu \mathrm{m} / \mathrm{s}$ and $l_{\mathrm{m}}=20$ - $40 \mu \mathrm{m}$, respectively. The amplitude $\Delta l_{y}$ of the vesicle with asymmetric tail in the $x y$ plane $(\mathrm{s} 2)$ is smaller than in $x z$ plane $(\mathrm{s} 1)$, since its tail is still at the wide part of the channel and elongated when the main body is at its narrowest part.

For vesicles, which flow in the center of the channel, similar symmetric shape oscillations are observed in the narrow and wide channels (in agreement with our predictions for fast flows from perturbation theory). However, when the vesicle is slightly displaced from the center $(y=0)$ in the wider channel it displays orientational oscillations, as shown in Fig. 6] Here, the asymmetry of the flow field acts to magnify the orientational oscillations, which are accompanied by strong shape oscillations between slipper and ellipsoidal shapes. Thus, a mixed state of orientational and shape oscillations appears for $\kappa^{*} \lesssim 1$; it appears to be long-lived due to the small lift force for wider channels.

Vesicles in sawtooth-shaped channels in slow flows. - Finally, we want to briefly discuss the dynamics of large vesicles at slow flows, with $\kappa^{*} \gg 1$. If the vesicle size is larger than the smallest channel width $L_{y}^{\min }$, vesicles can not flow through the channel without a shape deformation. Thus, with decreasing flow velocity, the dynamics changes from shape oscillations to a trapped state 36] (instead of the orientational oscillation). The transition velocity depends on $R_{\mathrm{S}} / L_{y}^{\min }, V^{*}$, and $\kappa^{*}$. A microchannel with a strong variation of channel width (for instance the channel in Fig. 6(a) of Ref. [14), is suitable for a study of this trapped-escape transition.

Summary. - We have studied the dynamics of vesicles in structured microchannels, both theoretically and experimentally. For large reduced volumes, the vesicles periodically change their shape (large flow rates) or their orientation (small flow rates), depending also on the channel geometry. For smaller reduced volumes, we find a novel shape with a long asymmetric tail. The good agreement of theoretical and experimental results shows that flow of vesicle suspensions in complex flow geometries can now be understood quantitatively.

$$
* * *
$$

We acknowledge support of this work through the DFG priority program "Nano- and Microfluidics" (SPP 1164) and the "Nanosystems Initiative Munich" (NIM).

\section{REFERENCES}

[1] Kantsler V. and Steinberg V., Phys. Rev. Lett., 96 (2006) 036001.

[2] Seifert U., Eur. Phys. J. B, 8 (1999) 405.

[3] Noguchi H. and Gompper G., Phys. Rev. Lett., 93 (2004) 258102.

[4] Noguchi H. and Gompper G., Phys. Rev. E, 72 (2005) 011901.

[5] Misbah C., Phys. Rev. Lett., 96 (2006) 028104.

[6] Noguchi H. and Gompper G., Phys. Rev. Lett., 98 (2007) 128103.

[7] Lebedev V. V., Turitsyn K. S. and Vergeles S. S., New. J. Phys., 10 (2008) 043044.

[8] Finken R., Lamura A., Seifert U. and Gompper G., Eur. Phys. J. E, 25 (2008) 309.

[9] Noguchi H., J. Phys. Soc. Jpn., 78 (2009) 041007.

[10] Higgins J. M., Eddington D. T., Bhatia S. N., and Mahadevan L., Proc. Natl. Acad. Sci. USA, 104 (2007) 20496.

[11] Whitesides G. M., Nature, 442 (2006) 368.

[12] Abrarian M., Faivre M., and Stone H. A., Proc. Natl. Acad. Sci. USA, 103 (2006) 538.

[13] Yang S., Uendar A. and Zahn J., Lab Chip, 6 (2006) 871.

[14] Abkarian M., Faivre M., and Stone H. A., Biomed. Mater., 3 (2008) 034011.

[15] Vitkova V., Mader M. and Podgorski T., Europhys. Lett., 68 (2004) 398.

[16] Tsukada K., Sekizuka E., Oshio C. and Minamitani H., Microvasc. Res., 61 (2001) 231.

[17] Pozrikidis C., Phys. Fluids, 17 (2005) 031503.

[18] Noguchi H. and Gompper G., Proc. Natl. Acad. Sci. USA, 102 (2005) 14159.

[19] Kantsler V., Segre E. and Steinberg V., Phys. Rev. Lett., 99 (2007) 178102.

[20] Turitsyn K. S. and Vergeles S. S., Phys. Rev. Lett., 100 (2008) 028103.

[21] Watanabe N., Kataoka H., Yasuda T. and Takatani S., Biophys. J., 91 (2006) 1984.

[22] Noguchi H., arXiv:0903.0038 [cond-mat.soft], (2009) .

[23] Noguchi H., J. Phys. Soc. Jpn., 79 (2010) in press.

[24] Angelova M. I., Giant Vesicles, edited by Luisi P. L. and Walde P. (Wiley, New York), sect. Liposome Electroformation, pp. 27-36 2000.

[25] Olla P., J. Phys. II, 7 (1997) 1533.

[26] Abrarian M., Lartigue C. and Viallat A., Phys. Rev. Lett., 88 (2002) 068103.

[27] Callens N. et al., EPL, 83 (2008) 24002.

[28] Coupier G., Kaoui B., Podgorski T. and Misbah C., Phys. Fluids, 20 (2008) 111702.

[29] Danker G., Vlahovska P. M. and Misbah C., Phys. Rev. Lett., 102 (2009) 148102.

[30] Xia Y. and Whitesides G. M., Angew. Chem. Int. Ed., 37 (1998) 550.

[31] Milner S. T. and Safran S. A., Phys. Rev. A, 36 (1987) 4371.

[32] Gompper G. and Kroll D. M., J. Phys. Condens. Matter, 9 (1997) 8795.

[33] Malevanets A. and Kapral R., J. Chem. Phys., 110 (1999) 8605.

[34] Kapral R., Adv. Chem. Phys., 140 (2008) 89. 
[35] Gompper G., Ihle T., Kroll D. M., and Winkler R. G., Adv. Polym. Sci., 221 (2009) 1.

[36] Gompper G. and Kroll D. M., Phys. Rev. E, 52 (1995) 4198. 